\title{
Common mistakes in the Dual-Energy X-ray Absorptiometry (DXA) in Turkey. A Retrospective Descriptive Multicenter Study
}

\author{
Ali Yavuz Karahan ${ }^{1, *}$, Bugra Kaya ${ }^{2}$, Banu Kuran $^{3}$, Ozlem Altındag $^{4}$, Pelin Yildirim ${ }^{5}$, Sevil Ceyhan Dogan', \\ Aynur Basaran', Ender Salbas ${ }^{7}$, Turgay Altınbilek, $^{8}$ Tuba Guler ${ }^{5}$, Sena Tolu', Zekiye Hasbek ${ }^{10}$, Banu Ordahan $^{1}$, \\ Ercan Kaydok $^{11}$, Ufuk Yucel ${ }^{11}$, Selcuk Yesilyurt ${ }^{12}$, Almula Demir Polat ${ }^{13}$, Murat Cubukcu ${ }^{14}$, Omer Nas ${ }^{15}$, \\ Umit Sarp $^{15}$, Ozan Yasar ${ }^{16}$, Seher Kucuksarac ${ }^{1}$, Gozde Turkoglu', Ahmet Karadag ${ }^{17}$, Sinan Bagcaci ${ }^{18}$, \\ Kemal Erol $^{19}$, Emel Guler ${ }^{20}$, Serpil Tuna ${ }^{21}$, Ahmet Yildirim ${ }^{22}$, Savas Karpuz' ${ }^{1}$
}

\footnotetext{
${ }^{1}$ Department of Physical Medicine and Rehabilitation, Beyhekim State Hospital of Konya Konya/Turkey

${ }^{2}$ Department of Nuclear Medicine of Necmettin Erbakan University, Meram Faculty of Medicine Konya/Turkey

${ }^{3}$ Department of Physical Medicine and Rehabilitation, Sisli Etfal Training and Research Hospital Istanbul/Turkey

${ }^{4}$ Department of Physical Medicine and Rehabilitation, Gaziantep University Sahinbey Research and Training Hospital, Gaziantep/Turkey

${ }^{5}$ Department of Physical Medicine and Rehabilitation, Derince Training and Research Hospital Kocaeli/Turkey

${ }^{6}$ Department of Physical Medicine and Rehabilitation, Cumhuriyet University, Faculty of Medicine Sivas/Turkey

${ }^{7}$ Department of Physical Medicine and Rehabilitation, State Hospital of Agri/Turkey

${ }^{8}$ Department of Physical Medicine and Rehabilitation, Physical therapy High school of Health Sciences of University of Halic, Istanbul/Turkey

${ }^{9}$ Department of Physical Medicine and Rehabilitation, Medipol University, Faculty of Medicine Istanbul/Turkey

${ }^{10}$ Department of Nuclear Medicine, Cumhuriyet University, Faculty of Medicine Sivas/Turkey

${ }^{11}$ Department of Physical Medicine and Rehabilitation, State Hospital of Nevsehir/Turkey

12. Department of Physical Medicine and Rehabilitation, Physical Medicine and Rehabilitation Hospital of Yoncali, Kutahya/ Turkey

${ }^{13}$ Department of Physical Medicine and Rehabilitation, State Hospital of Afyon/Turkey

${ }^{14}$ Department of Physical Medicine and Rehabilitation, State Hospital of Denizli/Turkey

${ }^{15}$ Department of Physical Medicine and Rehabilitation, State Hospital of Yozgat/Turkey

${ }^{16}$ Department of Physical Medicine and Rehabilitation, Amasya University Sabuncuoglu Serefeddin Research and Training Hospital, Amasya/Turkey

${ }^{17}$ Department of Physical Medicine and Rehabilitation, State Hospital of Sivas/Turkey

${ }^{18}$ Department of Physical Medicine and Rehabilitation, State Hospital of Hakkari/Turkey

${ }^{19}$ Department of Physical Medicine and Rehabilitation, State Hospital of Nigde/Turkey

${ }^{20}$ Department of Physical Medicine and Rehabilitation, Kayseri Training and Research Hospital Kayseri/Turkey

${ }^{21}$ Department of Physical Medicine and Rehabilitation, Akdeniz University, Faculty of Medicine Antalya/Turkey

${ }^{22}$ Department of Orthopedics and Traumatology, Beyhekim State Hospital of Konya Konya/Turkey

* Corresponding author: Department of Physical Medicine and Rehabilitation, State Hospital of Konya/Turkey, Yunusemremhnuranşskno1 meramkonya; e-mail: ayk222@hotmail.com
}

Summary: Background: Osteoporosis is a widespread metabolic bone disease representing a global public health problem currently affecting more than two hundred million people worldwide. The World Health Organization states that dual-energy X-ray absorptiometry (DXA) is the best densitometric technique for assessing bone mineral density (BMD). DXA provides an accurate diagnosis of osteoporosis, a good estimation of fracture risk, and is a useful tool for monitoring patients undergoing treatment. Common mistakes in BMD testing can be divided into four principal categories: 1) indication errors, 2) lack of quality control and calibration, 3) analysis and interpretation errors, and 4) inappropriate acquisition techniques. The aim of this retrospective multicenter descriptive study is to identify the common errors in the application of the DXA technique in Turkey. Methods: All DXA scans performed during the observation period were included in the study if the measurements of both, the lumbar spine and proximal femur were recorded. Forearm measurement, total body measurements, and measurements performed on children were excluded. Each examination was surveyed by 30 consultants from 20 different centers each informed and trained in the principles of and the standards for DXA scanning before the study. Results: A total of 3,212 DXA scan results from 20 different centers in 15 different Turkish cities were collected. The percentage of the discovered erroneous measurements varied from $10.5 \%$ to $65.5 \%$ in the lumbar spine and from $21.3 \%$ to $74.2 \%$ in the proximal femur. The overall error rate was found to be $31.8 \%(\mathrm{n}=1021)$ for the lumbar spine and $49.0 \%(\mathrm{n}=1576)$ for the 
proximal femur. Conclusion: In Turkey, DXA measurements of BMD have been in use for over 20 years, and examination processes continue to improve. There is no educational standard for operator training, and a lack of knowledge can lead to significant errors in the acquisition, analysis, and interpretation.

Keywords: Osteoporosis; Dlagnosis; Dual Energy X-ray Absorptiometry; Technician; Education

\section{Introduction}

Bone mineral density (BMD) is used in clinical practice as an indirect indicator of osteoporosis and fracture risk (1). Bone densitometry has quickly become the internationally accepted in-vivo bone mass measurement (1-3). The modalities of bone densitometry instruments include dual-energy X-ray absorptiometry (DXA), quantitative ultrasound, and quantitative computed tomography. DXA is realized as the reference technique to measure BMD in the lumbar spine, proximal femur, forearm, and whole body (2-4).

The World Health Organization (WHO) considers DXA to be the best densitometric technique for assessing BMD. DXA allows accurate diagnosis of osteoporosis, helps to determine the estimate fracture risk and monitoring of patients undergoing treatment (1-5). The primary target of DXA is to quantify BMD accurately and reproducibly and to compare that measurement with a reference population of asymptomatic individuals. Low measurement values on DXA predict the risk of fractures of the spine and hip, analogous to the relationship between high serum cholesterol and the risk of heart disease, or between high blood pressure and the risk of stroke. DXA is also useful in evaluating the effectiveness of FDA-approved therapies for osteoporosis, such as alendronate, risedronate, and raloxifene $(1,2,4-6)$.

Positive features of DXA include the safety of its performance, the short investigation time and the ease of use. A DXA measurement may take several minutes to complete with minimal radiation exposure. The densitometer produces ionizing radiation in the form of X-rays and uses laser radiation to position scans. However, the radiation exposure is so low that no shielding of rooms or health professionals is required. The radiation from a DXA scan is less than one would receive during a round trip cross-country airplane flight or during a day of normal background radiation $(4,7,8)$.

In measuring BMD, DXA provides a high degree of accuracy, although precision of DXA varies across operators and equipment. Many factors related to the equipment, the operator and the patient determine DXA precision. Operator-related factors are known to contribute to both long- and short-term precision errors. Differences in patient positioning and defining the regions of interest (ROI), both heavily operator dependent, contribute to these variations $(3,7-9)$. It is estimated that more than five thousand DXA instruments are in regular use worldwide and it is well known that the operators have had widely varied instruction and experi- ence. Operator training in different centers and countries is not standardized, and a lack of knowledge and skill can introduce errors in acquisition, analysis and interpretation of the scans $(6,7,10)$.

Common errors in BMD testing can be separated into four categories: 1) indication errors, 2) lack of quality control and calibration, 3) analysis and interpretation errors, and 4) acquisition errors (8-15). Acquisition errors have been well defined in previous studies including improper patient positioning, inappropriate scan mode, invalid skeletal site, persistent artifacts from the scanned area and incorrect demographic information (8-15). To our knowledge, there is no multicenter study that identifies and quantifies acquisition errors in DXA scanning in Turkey.

The aim of this retrospective descriptive multicenter survey is to identify common errors in the acquisition of DXA scanning in Turkey.

\section{Materials and Methods}

This descriptive study was conducted retrospectively over a period of 6 months from January 2014 to June 2014, in widely dispersed regions of Turkey. Data analysis and the study were approved by both the local scientific and ethical committees. We analyzed DXA scan results of 20 different centers in 15 different cities in Turkey (Figure 1). The project was approved by the Necmettin University Human Research Ethics Committee (NEUHREC) (Approval number: 2014/662) Individual ethics approval was also obtained from the (NEUHREC) responsible for each of the centers that participated in the project.

Consecutively performed DXA scan results were included the study if each contained a measurement of the lumbar spine and the proximal femur. All available scans performed over the observation period were considered. Forearm measurements, total body measurements or measurements carried out on children were excluded.

Studies were surveyed by 30 consultants from 22 different centers that were informed and trained on DXA scanning principles and standards before the study. Screening was performed according to the following guidelines:

For the lumbar spine (Figure 2a):

- The spine must be centered and straight (centered spinous processes).

- The scan must accurately show L1 through L5, as well as the ribs attached to T12 at the top of the view. 


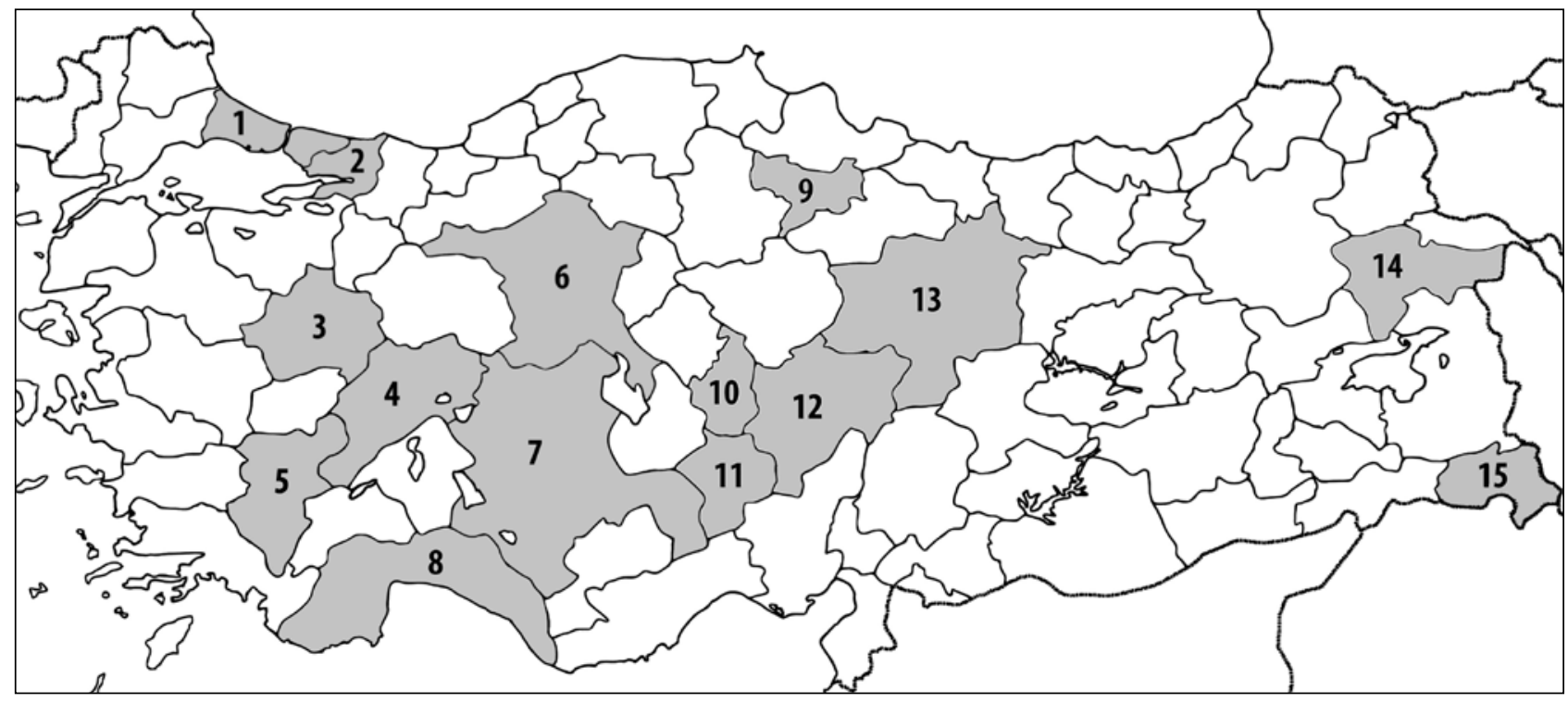

Fig. 1: Locations of DXA sites that contributed to the study: 1. Istanbul, 2. Kocaeli, 3. Kütahya, 4. Afyon, 5. Denizli, 6. Ankara, 7. Konya, 8. Antalya, 9. Amasya, 10. Nevşehir, 11. Niğde, 12. Kayseri, 13. Sivas, 14. Ağrı, 15. Hakkari.

- A small amount of the iliac crest must be visible in the lower corners of the view.

- Similar amounts of soft tissue must appear on each side of the entire spine.

- The region of interest (ROI) must be correctly oriented. ROI areas must include an adequate amount of soft tissue but must not include excessive ribs or iliac crests.

- Marking of vertebrae located in the ROI must be in the correct order.

- ROI must not contain artifacts or foreign bodies like metals, surgical clips, contrast medium, plastic materials, jewelry, body piercings, zippers or buttons.

- The scan must be free of distorting anatomies like laminectomy or spina bifida occulta.

- There must be no conditions that can affect the region being examined or invalidate the measurements like $\mathrm{Pa}$ get's disease, ankylosing spondylitis, aortic calcification, severe scoliosis or degenerative changes.

For the proximal femur (Figure $2 b$ ):

- The image must include the entire femoral head, neck, and approximately $7.5 \mathrm{~cm}$ (3 inches) of the femoral shaft.

- The femoral shaft must be aligned parallel to the long dimension of the measurement rectangle.

- The correct amount of soft tissue must be visible lateral to the greater trochanter.

- Internal rotation of the hip must be confirmed by little or no visible lesser trochanter.

- The preferred position for the femoral neck ROI differs depending on equipment manufacturers. The selection of the ROI (greater trochanter, proximal femoral neck or femoral head) must be correct.
- There must be no conditions that can affect the region being scanned or invalidate the measurements like fractures, callus formation or bladder stone.

Also, the specifics of the equipment and the department responsible for the DXA scanning for each institution were recorded.

Statistical analysis of data was performed using the computerized software program SPSS version 13 (SPSS, Inc., Chicago, IL, USA). Variables designed as some categorical (true or false) and derived variables (absolute and percent change) for the statistical analysis plan. Descriptive data were presented as mean \pm standard deviation. Demographic and clinical characteristics were compared using the chi-square test. Independent samples t test was used for the comparison of the two groups. A "p" value less than 0.05 was considered as statistically significant.

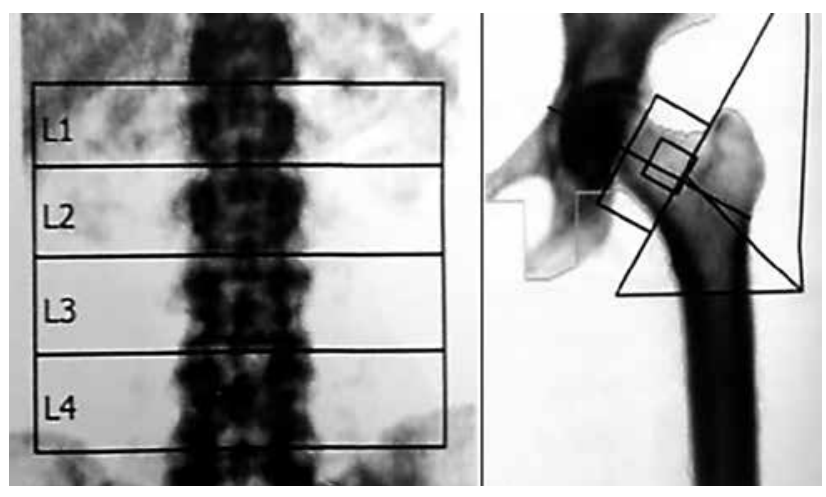

Fig. 2: Correct positioning in lumbar and proximal femur scanning. 


\section{Results}

A total of 3,212 DXA scan results were examined, acquired from 22 different centers in 15 different Turkish cities. Ten of the 22 centers were state hospitals, 8 were university hospitals, and 4 were training and research hospitals. The Radiology department was responsible for DXA scanning in 12 centers, and the Department of Nuclear Medicine was in charge in 10 centers.

According to our criteria, the percentage of unacceptable results ranged from $10.5 \%$ to $65.5 \%$ (average $31.7 \%$, $\mathrm{n}=1021$ ) for measurement of the lumbar spine and from $21.3 \%$ to $74.2 \%$ (average $49.0 \%, \mathrm{n}=1576$ ) in the proximal

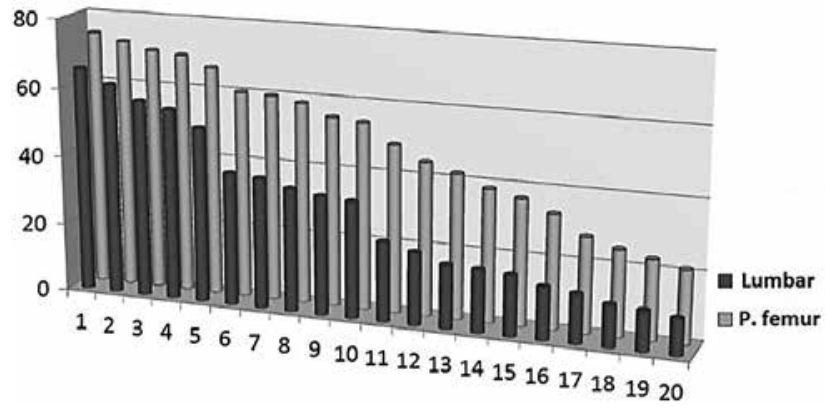

Fig. 3: Error rate in lumbar and proximal femur measurements in each of the 20 centers.
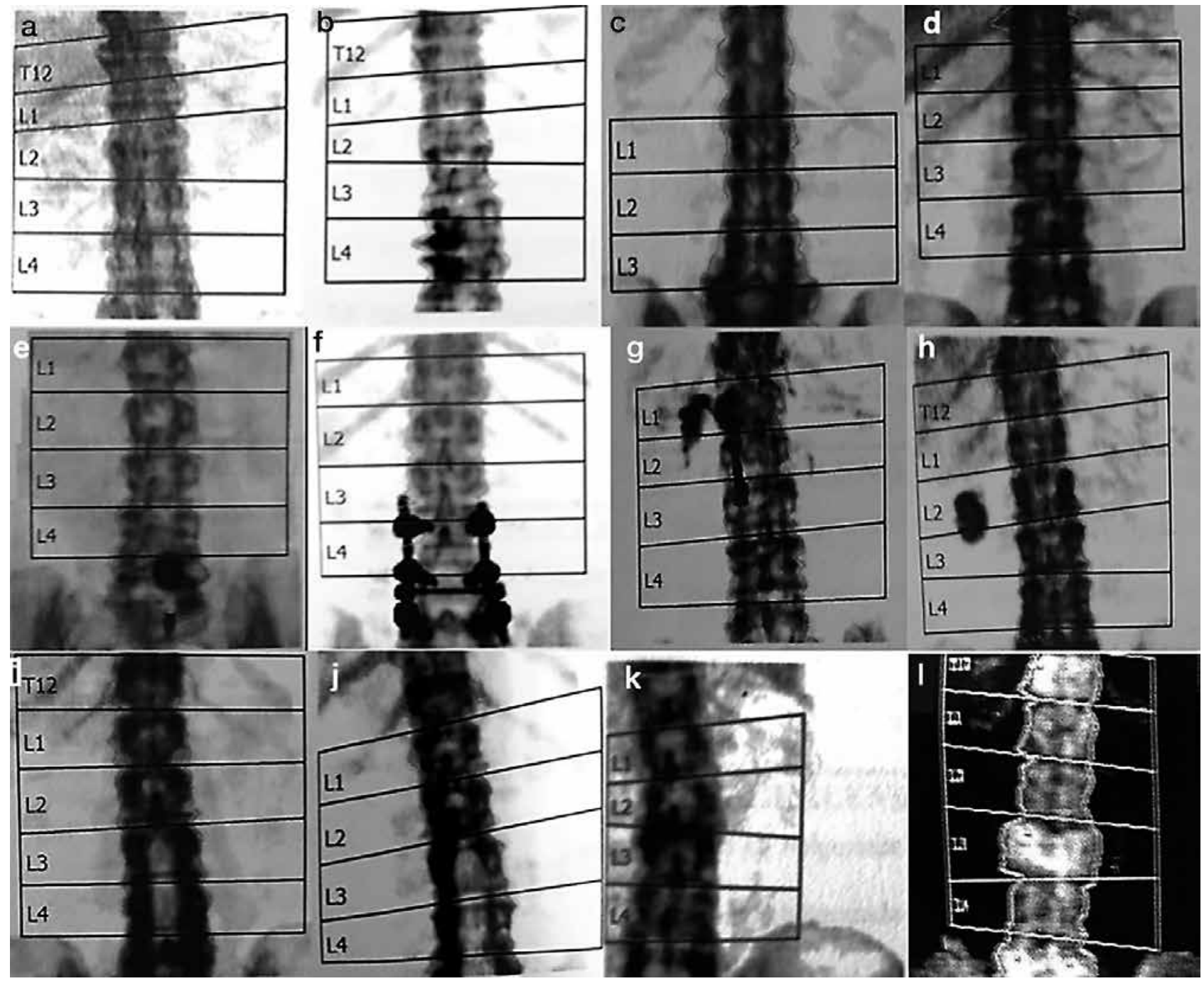

Fig. 4: Common sources of error for lumbar spine DXA measurements.

$\mathbf{a} / \mathbf{b}$ : Incorrect placing of the ROI (lines should not coincide with the vertebral bodies). $\mathbf{c} / \mathbf{d}$ : Region of interest must be marked in correct order. $\mathbf{e}-\mathbf{h}$ : Artifacts and foreign bodies (e. Button and zipper; f. Vertebral internal fixator; g. Calcification of the omentum and zipper; h. Gallstone). i/j: Laminectomy defects. k: Right-leaning ROI. I: Paget's disease. 

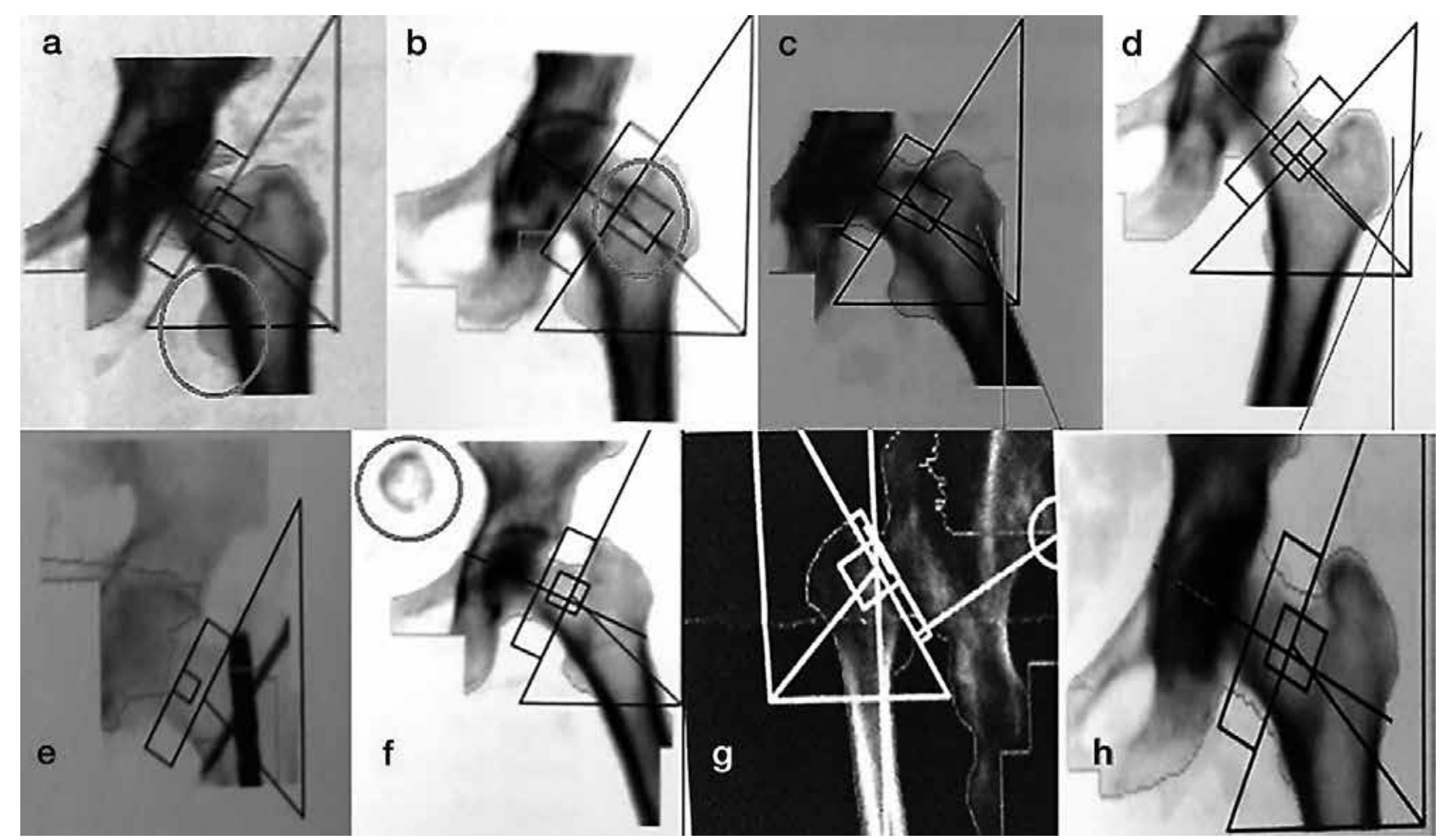

Fig. 5: Common sources of error for proximal femur measurements.

a: Inadequate internal rotation of hip (too much of the lesser trochanter is showing [circle]). b: Incorrect femoral neck region of interest placement (region of interest must be set on proximal femur neck). c: Exaggerated abduction of the hip. d: Exaggerated adduction of the hip. e-f: Artifacts or foreign bodies (e. Internal fixator in the proximal femur; f: Bladder calculus [circle]). g: Congenital hip dislocation. h: Inappropriately restricted and narrow scan area.

femur (Figure 3). Errors in defining the ROI was the most common error in lumbar spine results $(16.2 \%, \mathrm{n}=522)$. Other disqualifying problems included the presence of artifacts and foreign bodies, lateral misalignment of the ROI, laminectomy defects, Paget's disease, severe scoliosis and degenerative changes (Figure 4).

Inadequate internal rotation of hip was the most common disqualifying error in proximal femur measurements. Other errors included the faulty definition of the ROI, exaggerated adduction or abduction of the hip, artifacts or foreign bodies in the scanned area, congenital hip dislocation, and restricted or narrow scan area (Figure 5).

There were no statistically significant differences in errors between the state hospitals, the university hospitals or the training and research hospitals $(p>0.05)$. There also were no statistically significant differences between the radiology and the nuclear medicine departments responsible for the measurements $(p>0.05)$.

\section{Discussion}

The results of this study show seriously high error rates in DXA assessment of the lumbar spine (31.7\%) and the proximal femur $(49.0 \%)$ in 22 centers in Turkey. Different types of acquisition errors detected in both the lumbar and proximal femur DXA scans were responsible for the mistakes. Data were collected from three classes of hospitals, state, university, and training and research hospitals between which there were no significant differences.

DXA is a clinically proven technique of measuring BMD in the lumbar spine, proximal femur, forearm, and whole body. With DXA, it is possible to measure BMD accurately and reproducibly and to compare that measurement to a reference population of asymptomatic individuals (1-3). Therefore, in the diagnosis and management of osteoporosis and in determining future fracture risk, DXA is the most frequently used method. Some of the challenges in performing reliable DXA measurements include differences in equipment, acquisition techniques, reference databases, reporting methods, and descriptive terminology $(7,8,10)$. Much of the responsibility of DXA fall to the operator such as reviewing the patient's skeletal health history, entering demographic data into the computer, performing the image acquisition, and analyzing the scan $(8,10,13)$. The operator must evaluate and, if necessary, adjust the computer-selected bone edges and markers of bone ROI. Also, hip bone edges and ROI must be correct, with particular attention paid to the location of the femoral neck box $(7-11,13,16)$. Therefore, 
DXA clearly is an operator-dependent method. This situation marks the crucial need for education and experience to perform high-quality DXA.

The International Society for Clinical Densitometry (ISCD) is a society with a mission to 1) enhance knowledge, skill, and quality of densitometry among healthcare professionals, 2) educate and certify clinicians and technologists, 3 ) increase patient awareness, 4) improve access to densitometry, 5) support clinical and scientific advances in the field, and 6) foster the exchange of scientific information. To address these issues, the ISCD periodically holds Position Development Conferences (1, 5, 9). Many guidelines and studies have drawn attention to frequent errors in the clinical use of DXA. Also, there are two available Turkish DXA guidelines $(17,18)$. However, to our knowledge, no detailed quality assessment and error flagging study of DXA bone mineral density measurement has been carried out across this country. The present study is the first one.

The selection of the ROI can be a major source of error even for experienced operators, especially in roto-scoliosis of the lumbar spine $(7,16,19)$. In a typical DXA evaluation, there is usually a gradual increase in vertebral area progressing from L2 to L4. If this is not evident in the results, it is necessary to check the selection of ROI levels $(7,16,19)$. In our study, ROI definition errors were the most common problem in lumbar spine measurements (Figure $4 \mathrm{a}-\mathrm{d}$ ).

Kendler et al.(14) used undissolved calcium (Ca) as an artifact to detect the effect on DXA results. They placed the $\mathrm{Ca}$ tablets in the soft tissue field, overlying bone and overlapping both bone and soft tissue. An overlying Ca tablet had a considerable effect on $\mathrm{BMD}$, resulting in $12.6 \%$ increase in the density of a single vertebral body (14). The Ca tablet artifact showcases the importance of other spine DXA artifacts on imprecision (14). Lumbar spine osteophytes explained $22 \%$ of the variation in spine BMD in men and $17 \%$ in women. Artifacts such as mal-positioning, osteophytes, and aortic calcification, which could affect BMD, could be seen in the Ca tablet trials $(1,17,20)$. In our study, we encountered different types of artifacts such as buttons, zippers, gallstones or nonspecific omental calcification (Figure $4 \mathrm{e}-\mathrm{h}$ ). Abnormal densities are obtained in Paget's disease because of the larger and denser vertebrae. An overlying calcified aorta may raise apparent vertebral density. In addition, absent bony structures, as in laminectomy or spina bifida, or displaced bone as in vertebral rotation due to idiopathic scoliosis could decrease the BMD estimation.

The BMD response following anti-resorptive or anabolic therapy is greater in the lumbar spine than in the proximal femur. However, this progress could be obscured in the presence of degenerative skeletal conditions or by the internal fixation of the lumbar spine. Correct positioning of the patient during the measurement of proximal femur BMD is also vital to obtaining precise values $(15,21)$. According to cadaveric studies, the minimum BMD in the femoral neck was recorded when the femoral neck was parallel to the examination surface, and gradual increase in BMD occurred when the femur was rotated either internally or externally $(22,23)$. Therefore, accurate estimation of BMD requires that the axis of the femoral neck be maintained parallel to the inspection surface and perpendicular to the X-ray beam. DXA equipment manufacturers provide different types of positioning aids to achieve this objective. Girard et al. (22) found a significant change in femoral neck BMD when the leg was rotated $10-15^{\circ}$ from its neutral position. But Lekamwasam S. et al. (21) examined the effect of leg rotation by 10 degrees either internally or externally on hip BMD in living subjects and concluded that their results show the effect of mal-positioning of the hip during DXA scanning were more significant in longitudinal than in cross-sectional BMD analysis (21). According to our results, inadequate internal rotation of hip was the most common error in proximal femur results. Definition errors of the ROI, excessive adduction or abduction of the hip, artifacts, foreign bodies, congenital hip dislocation, and inappropriate scan area were the other sources of error (Figure 5).

DXA estimates of BMD have been in use for over 20 years in Turkey, and examination protocols continue to improve results (17). There is no educational standard for operator training, and a significant lack of knowledge can lead to flaws in an acquisition, analysis, and interpretation of the scan. Clinical and technical awareness of potential sources of error and artifact are pivotal to maximize the value of DXA measurements of BMD.

\section{Limitations of study}

The existing data was gleaned from records of 22 center from 15 provinces of Turkey. Turkey has 81 provinces, and our results that obtained from this study could not generalize to the generalize to the whole country. Although precision errors for DXA scanning evaluated for this study, we don't know the full effects of these errors to the results and how these incorrect measurements effects on the clinical decisions. But this retrospective study helps to focus the question of "frequencies and definitions of common mistakes in the DXA for Turkey" and determines an appropriate sample size. A particularly useful application of this retrospective study is a pilot study that should be completed in anticipation of a prospective trial.

\section{Acknowledgements}

Only personal funds were used for this study. No external funds were obtained. Financial disclosure statements have been achieved, and no conflicts of interest have been reported by the authors.

\section{References}

1. Sindel D, Gula G. Assessment of Bone Mineral Density in Osteoporosis. Türk Osteoporoz Dergisi 2015; 21: 23-9.

2. Tunç G, Doğan SC, Hizmetli S, Hayta E. In urban areas of Sivas city the determination of bone mineral density reference values of healthy women who admitted to 
Cumhuriyet University Hospital Physical Medicine and Rehabilitation outpatient clinic. Türk Osteoporoz Dergisi 2014; 20: 104-9.

3. Sindel D. Osteoporozda görüntüleme yöntemlerinde gelişmeler. Türkiye Klinikler Journal of Physical Medicine Rehabilitation Special Topics 2009; 2(1): 50-61

4. Aslan A, Uysal E, Karakoyun Ö. Bone Mineral Density Value in Kastamonu and Area of Turkish Society Women J Clin Anal Med 2013; 4(3): 209-12.

5. Guglielmi G, Ferrari F, Bazzocchi A. Bone Mineral Density and Quantitative Imaging. In: W. C. G. Peh ed. Pitfalls in Diagnostic Radiology. Springer, 2015: 109-32

6. Allin S, Munce S, Carlin L, Butt D, Tu K, Hawker G, et al. Fracture risk assessmen after BMD examination: whose job is it, anyway? Osteoporosis International 2014 25(5): 1445-53.

7. Lewiecki EM, Lane NE. Common mistakes in the clinical use of bone mineral density testing. Nature clinical practice Rheumatology 2008; 4(12): 667-74

8. El Maghraoui A, Roux C. DXA scanning in clinical practice. QJM: monthly journal of the Association of Physicians 2008; 101(8): 605-17.

9. Guglielmi G, Diano D, Ponti F, Bazzocchi A. Quality assurance in bone densitometry. Current Radiology Reports 2014; 2(2): 1-6.

10. Khan AA, Colquhoun A, Hanley DA, Jankowski LG, Josse RG, Kendler DL, et al. Standards and guidelines for technologists performing central dual-energy $\mathrm{X}$-ray absorptiometry. Journal of clinical densitometry: the official journal of the International Society for Clinical Densitometry 2007; 10(2): 189-95.

11. Messina C, Bandirali M, Sconfienza LM, D’Alonzo NK, Di Leo G, Papini GD, et al. Prevalence and type of errors in dual-energy $\mathrm{x}$-ray absorptiometry. European radiology 2015; 25(5): 1504-11.

12. Ott SM, Ichikawa LE, LaCroix AZ, Scholes D. Navel jewelry artifacts and intravertebral variation in spine bone densitometry in adolescents and young women. Journal of clinical densitometry: the official journal of the International Society for Clinical Densitometry 2009; 12(1): 84-8.

13. Lewiecki EM, Binkley N, Petak SM. DXA quality matters. Journal of clinical densitometry: the official journal of the International Society for Clinical Densitometry 2006; 9(4): 388-92.

14. Kendler DL, Kiebzak GM, Ambrose CG, Dinu C, Robertson S, Schmeer P, et al Effect of calcium tablets on interpretation of lumbar spine DXA scans. Journal of clinical densitometry: the official journal of the International Society for Clinical Densitometry 2006; 9(1): 97-104.

15. Wong JCH, Ong B. Evaluation of Femur Angle Abduction/Adduction and Bone Mineral Density Values. Journal of Clinical Densitometry 2005; 8(4): 472-5.

16. Vasić J, Gojković F, Zvekić-Svorcan J, Ćulafić-Vojinović V, Elez J, Filipović K. The most common mistakes in bone mineral density testing with DXA method. MD-Medical data 2013; 5(3): 271-8.

17. Manisali M, Ozaksoy D, Dogan S. Osteoporozda Radyolojik Görüntüleme. Turkiye Klinikleri Journal of Orthopaedics and Traumatology Special Topics 2010; 3(2): $29-38$.

18. Erselcan T, Ozen A, Yuksel D, Altun D, Ozturk E, Balci TA, et al. Kemik mineral yoğunluğu ölçümü uygulama kılavuzu. Turk J Nucl Med 2009; 18(1): $31-40$.

19. Krueger D, Vallarta-Ast N, Libber J, Checovich M, Gangnon R, Binkley N. Positioner and clothing artifact can affect one-third radius bone mineral density measurement. Journal of clinical densitometry: the official journal of the International Society for Clinical Densitometry 2013; 16(2): 154-9.

20. Richmond B. DXAscanning to diagnose osteoporosis: Do you know what the results mean? Cleveland Clinic Journal of Medicine 2003; 20(4): 353-60.

21. Lekamwasam S, Lenora RS. Effect of leg rotation on hip bone mineral density measurements. Journal of clinical densitometry: the official journal of the International Society for Clinical Densitometry 2003; 6(4): 331-6.

22. Girard MS, Sartoris DJ, Moscona AA, Ramos E. Measured femoral density by dual-energy X-ray absorptiometry as a function of rotation. Orthopaedic review 1994; 23(1): 38-40.

23. Cheng XG, Nicholson PH, Boonen S, Brys P, Lowet G, Nijs J, et al. Effects of anteversion on femoral bone mineral density and geometry measured by dual energy X-ray absorptiometry: a cadaver study. Bone 1997; 21(1): 113-7.

Received: 23/05/2016

Accepted: 29/08/2016 